\title{
Undergraduate and postgraduate medical education on the prevention and control of healthcare-associated infection. More progress is needed
}

\author{
H Humphreys ${ }^{1,2}$, J Richards ${ }^{3,4}$ \\ 1. Department of Clinical Microbiology, The Royal College of Surgeons in Ireland, \\ 2. Department of Microbiology, Beaumont Hospital, Dublin, Ireland \\ 3. Department of Microbiology, Norfolk and Norwich University Hospital \\ 4. Faculty of Medicine, University of East Anglia, UK
}

doi: 10.3396/ijic.V7i2.012.11

\begin{abstract}
The prevention and control of healthcare-associated infection (HCAI) is a significant patient safety and quality issue that has not been accorded sufficient emphasis in the medical school curriculum. Compliance amongst medical students and graduates with basic measures such as hand hygiene is inferior to that amongst other healthcare groups such as nurses. While there are some studies assessing the knowledge of medical students on HCAI prevention, few have assessed attitudes and practice or behaviour. Although, HCAl is being recognised in the medical curriculum there is scope for greater co-ordination in educational delivery and assessment. At postgraduate level, there has been some success in enhancing the prevention of catheter-associated bloodstream infection through a multi-modal approach that has been shown to be superior to traditional measures. Education programmes need to be developed at the postgraduate level geared to the specialty, but much more work needs to be done to highlight the importance of this area and to ensure better compliance
\end{abstract}

Key words

hand hygiene, medical education, knowledge, attitudes, behaviours, device-related infections

\section{Corresponding author}

Hilary Humphreys Department of Clinical Microbiology,

RCSI Education and Research Centre, Beaumont Hospital, PO Box 9063, Dublin 9, Ireland

Phone +3531 8093708/3710, Fax +3531 8093709, Email hhumphreys@rcsi.ie 


\section{Introduction}

The prevention and control of healthcare-associated infection (HCAI) has traditionally not been a high priority in undergraduate and postgraduate medical education programmes. Where it has been covered, this has often been under related areas, e.g. surgical scrub, rather than as an important topic of infection prevention per se. More recently, with changes in the medical curricula in many developed countries, such as in the UK, there has been a move away from traditional disciplines such as microbiology to a more theme-based approach. Consequently, traditional teaching and learning in the area of microbiology and infection may not be as clearly identifiable in some courses as it was previously. The introduction of a systems-based, problem-orientated and team-based medical curriculum provides a significant opportunity for advancement in the education, training and promotion of healthcare-associated infection amongst medical students, under the headings or themes of professionalism and patient safety.

In order to ensure that the doctors of the future are safe practitioners, it is essential that they have the relevant knowledge and that this informs their attitude leading to appropriate professional behaviour. Compared with other healthcare professionals, medical doctors are less compliant in basic aspects of infection prevention and control, e.g. hand hygiene, compared with other healthcare professionals such as nurses, physiotherapists, etc. Consequently, there is a need to improve the education and learning of medical students worldwide.

\section{Undergraduate medical education}

While there are many studies and interventions that have assessed the knowledge base of medical students, there is a much smaller body of literature on attitudes and behaviours. For example, in a French study of 350 students, including 107 medical students, nursing students had a better overall score compared to medical students in the knowledge of hand hygiene, standard precautions and nosocomial infections. ${ }^{1}$

In Birmingham, UK, medical students were assessed for their knowledge of certain aspects of HCAI, of which $48 \%$ participated. ${ }^{2}$ This study assessed knowledge of hand hygiene, the colour of clinical waste bags, the use of gloves, the disposal of sharps, venepuncture and needle stick injuries. Just under half felt that not enough emphasis was placed on infection prevention and control, 50\% knew when they should return to the wards after being ill with diarrhoea and $83 \%$ knew what action to take in the event of a needle stick injury. ${ }^{2}$ However, given the developments in the UK and the greater emphasis politically and otherwise on the prevention and control of infection such as methicillin-resistant Staphylococcus aureus (MRSA) in the interim, it is possible that the results would be improved if repeated now. Again, in an assessment of knowledge of nearly 500 medical students in Iran, scores were approximately $66 \%{ }^{3}$

Practice rather than knowledge alone is a better assessment of on-going behaviour and in a study of medical students carrying out their final medical exams, only $9 \%$ of students used an alcohol hand gel on day 1 , before there was a notice instructing this, compared with $27 \%$ after a notice had been put up in the examination area on day $2 .{ }^{4}$ Forty per cent of these medical students felt that their compliance with hand hygiene was at least $80 \%$, whereas observed practice indicated otherwise. ${ }^{4}$

Education and learning can take many forms and the single traditional lecture has little impact. In a randomised controlled trial on the impact of a single lecture and video to evaluate knowledge amongst medical students on bloodborne viruses interventions in China, there was no difference between the baseline and post intervention periods in terms of knowledge of bloodborne pathogens. ${ }^{5}$ Many medical students and others learn by example and in particular through the influence of mentors or those senior personnel whom the student admires. In a study of nursing students, a mentor's practice of hand hygiene was the strongest predictor of the student rate of hand hygiene but glove use was also associated with increased hand hygiene rates. ${ }^{6}$

Fear or a wish to protect oneself is also a motivational factor in taking measures to prevent and control HCAI. This is well illustrated in a study of hand hygiene practice in Hong Kong before and during the SARS epidemic. The proportion of students who washed their hands before examining a patient increased from 
$33 \%$ to $60 \%$ between 2003 and 2004 as did those after examining the patient, i.e. increasing from $73 \%$ to $100 \% .{ }^{7}$ It would be interesting to review compliance now and see whether the impact of SARS locally has led to a permanent change in hand hygiene practice, or if this was merely a manifestation of the circumstances at the time.

In a Greek study of medical and nursing students, nursing students scored higher overall in terms of knowledge but the delivery of education on hand hygiene was different between medical and nursing students. ${ }^{8}$ Nursing students had more lectures, tutorials and lecture notes compared to the medical students, and there was a greater use of published guidelines, posters and the internet amongst nursing students. ${ }^{8}$

All the above clearly demonstrate that there is an educational deficit amongst medical students. Curriculum change has to address this to ensure that patient safety is prioritised. A survey of 31 of $38(82 \%)$ medical schools in the UK and the Republic of Ireland (Rol) revealed that HCAI as a quality and safety issue was covered in only $60 \%$ of medical schools but that waste, personal equipment and standard precautions were assessed in $60-67 \%$ of medical schools. ${ }^{9}$ There is also recognition of the need to improve programmes in this area in other parts of the world. In Thailand, none of 12 medical school directors surveyed expressed themselves satisfied with the teaching on HCAI but the survey itself subsequently resulted in modifications in two of these. ${ }^{10}$

\section{Postgraduate medical education}

Life-long learning is essential to ensure optimal practices in the prevention and control of HCAl. A number of surveys have indicated deficiencies in the practices of postgraduate medical doctors which may reflect inadequate knowledge. In one study despite presentations, text messages, informal teaching sessions, etc, these interventions did not result in any great improvement in the knowledge of non-consultant doctors in a number of areas including MRSA, the management of Clostridium difficile and vancomycinresistant enterococci, with $33 \%$ of medical doctors unaware that patients without diarrhoea were no longer infectious. ${ }^{11}$
It is recognised that in specialist areas such as intensive care, knowledge about key aspects of infection may be better as the healthcare professionals are confronted with the issues on a day-to-day basis as part of their practice. In an evaluation of the knowledge of 10 physicians, 41 nurses and eight respiratory therapists working in an intensive care unit, the mean total scores were approximately $80 \%$, with no significant differences between them. ${ }^{12}$ Surprisingly, respondents had the least knowledge about the frequency of humidifier changes and the optimal frequency of ventilator circuit changes.

The needs of nurses and doctors will also differ according to their particular responsibilities and training, e.g. doctors take the lead in active interventions to treat patients with infection, including starting antibiotics. This is reflected in a study from Dundee, UK where knowledge of the complications of MRSA and the antibiotic management of MRSA were superior amongst medical doctors compared with nurses. ${ }^{13}$

Device-related infections are potentially preventable and consequently there is much emphasis on the use of healthcare bundles to reduce these infections in recent years. Improvements in reducing infections and in improving professional practice have been seen in a number of recent studies that involved education programmes. ${ }^{14-17}$ Audiovisual education was an effective tool for practice change, resulting in a significant reduction in catheter-related bloodstream infections (CRBSI) in a neonatal intensive care unit. ${ }^{14}$ Multi-faceted education programmes on hand hygiene improved hand hygiene compliance before patient contact in a Dutch study. ${ }^{15}$ However, sustained interventions were required to maintain and improve strategies to reduce CRBSI in Brazil. ${ }^{16}$

Many medical doctors have been trained in the past to carry out some procedures based on the traditional approach of "observe one, do one and teach one." In a US study from Illinois, a simulation-based learning programme incorporating a 27-item skills checklist significantly reduced the incidence of CRBSI. This improvement was significantly better than the traditional education approach which was observation at the bedside. ${ }^{17}$ In some facets of HCAI prevention and 
control, interventions are required at different stages and these may vary from reminders to introducing blocks in a patient course or journey unless a particular intervention has been complied with. In an effort to improve prophylactic antibiotic use, a number of measures were required to improve compliance from $55 \%$ to $95 \%$, the final aspect of which was mandatory administration by an anaesthetist. ${ }^{18}$

\section{Conclusions}

It is clear that much work needs to be done to enhance and improve the education of medical students and doctors following graduation to improve practice and behaviour. Presently, in many medical schools around the world, this area is not adequately prioritised and there is insufficient assessment of practice and attitudes that would emphasise its importance. There is scope for the provision of materials that could be widely used and that would underpin many international initiatives such as the WHO campaign on hand hygiene. In the survey conducted in the UK and Rol, lectures, cases and practical demonstrations were considered useful by more than $90 \%$ of medical schools and $83 \%$ indicated that they would be willing to share a common pool of educational resources. ${ }^{9}$

While there is a commonality of approach amongst the surveyed medical schools it is clear that there is a lack of focus of what is important and efficient in terms of the delivery of teaching materials and their assessment. Increasingly various aspects of medical education are being delivered online or through webbased programmes. However, in resource-challenged settings, this may be difficult. A survey of English language medical schools in Africa of computer facilities showed poor student: computer ratios and although respondents were generally enthusiastic about the role of computers, there were difficulties with regard to computer speed and access to DVDRoms. ${ }^{19}$

In the postgraduate arena, the degree to which HCAI control and prevention is addressed depends on the speciality and probably the enthusiasm and interest of the individuals involved. In the United States, a survey of programme directors of residency programmes, indicated amongst other things, that HCAI prevention was covered under a general discussion of hospital policies or safety procedures during orientation. ${ }^{20}$ It thus appears that many programmes relegate this important issue into a general lecture without any subsequent reenforcement or without highlighting the importance of this area per se. A review of patient programmes and studies amongst healthcare professions has recently indicated the absence of an educational strategy for surgical trainees and this requires attention given the frequency and importance of surgical infections. ${ }^{21}$ As patients themselves become more educated about the risks and the consequences of $\mathrm{HCAl}$ and the measures that have been shown to be successful in their prevention, this will impact on postgraduate training programmes necessitating greater emphasis on education in this area.

While infection prevention and control practitioners are to the forefront in promoting education, until this is recognised as mandatory for all medical students and all doctors on an on-going basis, it is unlikely to become a general feature of postgraduate programmes. Consequently, we must strive to educate our colleagues on the importance of this area by improving both undergraduate and postgraduate medical education to ultimately enhance the quality and safety of patient care.

\section{References}

1. Tavolacci M-P, Ladner J, Bailly L, Merle V, Pitrou I, Czernichow P. Prevention of nosocomial infection and standard precautions: knowledge and source of information among healthcare students. Infect Control and Hosp Infect 2008; 29: 642-647.

2. Mann CM, Wood A. How much do medical students know about infection control? I Hosp Infect 2006; 64: 366-370.

3. Askarian M, Aramesh K, Palenik CJ. Knowledge, attitude, and practice toward contact isolation precautions among medical students in Shiraz, Iran. Am / Infect Control 2006; 34: 593596.

4. Hunt DCE, Mohammudally A, Stone SP, Dacre J. Handhygiene behaviour, attitudes and beliefs in first year clinical medical students. J Hosp Infect 2005; 59: 371-373.

5. Zhang Z, Yamamoto T, Wu XN, Moji K, Cai GX, Kuroiwa C. Educational intervention for preventing bloodborne infection among medical students in China. I Hosp Infect 2010; 75: 47-51.

6. Snow M, White GL, Alder SC, Stanford JB. Mentor's hand hygiene practices influence student's hand hygiene rates. Am J Infect Control 2006; 34: 18-24.

7. Wong T-W, Tam WW-S. Handwashing practice and the use of personal protective equipment among medical students after the SARS epidemic in Hong Kong. Am J Infect Control 2005; 33: $580-586$.

8. van de Mortel TF, Apostolopoulou E, Petrikkos G. A 
comparison of the hand hygiene knowledge, beliefs, and practices of Greek nursing and medical students. Am J Infect Control 2010; 38: 75-77.

9. O'Brien D, Richards J, Walton KE, Phillips MGA, Humphreys $\mathrm{H}$. Survey of teaching/learning of healthcare-associated infections in UK and Irish Medical schools. J Hosp Infect 2009; 73: 171-175.

10. Danchaivijitr $S$, Chakpaiwong $S$, Jaturatramrong $U$, Wachiraporntip A, Cherdrungsi R, Sripalakij S. Program on nosocomial infection in the curricula of medicine, dentistry, nursing and medical technology in Thailand. I Med Assoc Thai 2005; 88 (Suppl 10): S150-S154.

11. Fleming $\mathrm{M}$, Thomas $\mathrm{T}$, Humphreys $\mathrm{H}$. Educating junior doctors on healthcare-associated infection: more work to be done. I Hosp Infect 2010; 74(3): 302-304.

12. El-Khatib M, Zeineldine S, Ayoub C, Husari A, Bou-Khalil PK. Critical care clinicians' knowledge of evidence-based guidelines for preventing ventilator-associated pneumonia. Am J Crit Care 2010; 19: 272-277.

13. Easton PM, Sarma A, Williams FLR, Marwick CA, Phillips G, Nathwani D. Infection control and management of MRSA: assessing the knowledge of staff in an acute hospital setting. J Hosp Infect 2007; 66: 29-33.

14. Sannoh S, Clones B, Munoz J, Montecalvo M, Parvez B. A multimodal approach to central venous catheter hub care can decrease catheter-related bloodstream infection. Am J Infect Control 2010; 38: 424-429.
15. Helder OK, Brug J, Looman CWN, van Goudoever JB, Kornelisse RF. The impact of an education program on hand hygiene compliance and nosocomial infection incidence in an urban Neonatal Intensive Care Unit: An intervention study with before and after comparison. Int / Nurs Studies 2010; 47: 1245-1252.

16. Lobo RD, Levin AS, Oliveira MS, et al. Evaluation of interventions to reduce catheter-associated bloodstream infection: Continued tailored education versus one basic lecture. Am J Infect Control 2010; 38: 440-448.

17. Barsuk JH, Cohen ER, Feinglass J, McGaghie WC, Wayne DB. Use of stimulation-based education to reduce catheter-related bloodstream infections. Arch Intern Med 2009; 169: 14201423.

18. Whitman G, Cowell V, Parris K, et al. C. Prophylactic antibiotic use: Hardwiring of physician behaviour, not education, leads to compliance. J Am Coll Surg 2008; 207: 88-94.

19. Williams CD, Pitchforth EL, O'Callaghan C. Computers, the internet and medical education in Africa. Medical Education 2010; 44: 485-488.

20. Sahud AG, Bhanot N, Bhat S, Lane S, Bajwa R. Infection prevention education: are we neglecting it? Infect Control \& Hosp Epid 2010; 31: 199-201.

21. McHugh SM, Hill ADK, Humphreys H. Preventing healthcareassociated infection through education: Have surgeons been overlooked? The Surgeon 2010; 8: 96-100. 